\title{
Plasma-derived parasitic microRNAs have insufficient concentrations to be used as diagnostic biomarker for detection of Onchocerca volvulus infection or treatment monitoring using LNA-based RT-qPCR
}

\author{
Ole Lagatie $^{1} \cdot$ Linda Batsa Debrah $^{2} \cdot$ Alex Debrah $^{3} \cdot$ Lieven J. Stuyver $^{1}$
}

Received: 25 August 2016 / Accepted: 16 January 2017 / Published online: 22 January 2017

(C) The Author(s) 2017. This article is published with open access at Springerlink.com

\begin{abstract}
River blindness, caused by infection with the filarial nematode Onchocerca volvulus, is a neglected tropical disease affecting millions of people. There is a clear need for diagnostic tools capable of identifying infected patients, but that can also be used for monitoring disease progression and treatment efficacy. Plasma-derived parasitic microRNAs have been suggested as potential candidates for such diagnostic tools. We have investigated whether these parasitic microRNAs are present in sufficient quantity in plasma of Onchocerca-infected patients to be used as a diagnostic biomarker for detection of $O$. volvulus infection or treatment monitoring. Plasma samples were collected from different sources (23 nodulepositive individuals and 20 microfilaridermic individuals), microRNAs (miRNAs) were extracted using Qiagen miRNeasy kit, and a set of 17 parasitic miRNAs was evaluated on these miRNA extracts using miRCURY Locked Nucleic Acid (LNA) Universal RT microRNA PCR system. Of the 17 miRNAs evaluated, only 7 miRNAs were found to show detectable signal in a number of samples: bma-miR-236-1, bma-miR-71, ov-miR71-22nt, ov-miR71-23nt, ov-miR-100d, ov-bantam-a, and ov-miR-87-3p. Subsequent melting curve analysis, however, indicated that
\end{abstract}

Ole Lagatie

olagatie@its.jnj.com

1 Janssen Diagnostics, Janssen R\&D, Turnhoutseweg 30, 2340 Beerse, Belgium

2 Kumasi Centre for Collaborative Research, Kwame Nkrumah University of Science and Technology, Kumasi, Ghana

3 Faculty of Allied Health Sciences, Kwame Nkrumah University of Science and Technology, Kumasi, Ghana the signals observed for ov-miR-71 variants and ov-miR87-3p are non-specific. The other miRNAs only showed positive signal in one or few samples with $\mathrm{Cq}$ values just below the cutoff. Our data indicate that parasitic miRNAs are not present in circulation at a sufficiently high level to be used as biomarker for $O$. volvulus infection or treatment monitoring using LNA-based RT-qPCR analysis.

Keywords Onchocerca volvulus · River blindness · Onchocerciasis $\cdot$ Biomarker $\cdot$ miRNA $\cdot$ Diagnostic

\section{Introduction}

Onchocerciasis, a neglected tropical disease commonly known as river blindness, is caused by infection with the filarial nematode, Onchocerca volvulus (O. volvulus). In Africa, at least 120 million people are at risk of infection (Borup et al. 2003; Enk 2006). The last comprehensive survey conducted in 2008 indicated that 26 million people were infected with Onchocerca, of whom 265,000 were blind and 746,000 were visually impaired. In addition, approximately 4 million people suffer from onchodermatitis with severe itching (WHO 2010). Since 1987, ivermectin has been used in mass drug administration (MDA) programs to treat hundreds of millions of people resulting in a reduction of both visual impairment and symptomatic onchodermatitis (Cupp et al. 2011).

Besides clinical examination by palpation of nodules formed by adult worms (macrofilariae), diagnostic tools for detection of $O$. volvulus infection traditionally were limited to detection of microfilariae (mf) in small, superficial skin biopsy samples (socalled skin snips) (Taylor et al. 1989). More recent research, however, also resulted in the development of rapid-format tests for the detection of IgG4 antibodies to the parasitic antigen Ov16 (Chandrashekar et al. 1996; Golden et al. 2013; Lavebratt 
et al. 1994; Lipner et al. 2006; Steel et al. 2015; Weil et al. 2000). This test is predominantly useful in a surveillance setting where it can be used for the identification of incident infections in communities having already undergone MDA (Golden et al. 2013). Diagnostic tests that can distinguish between past and active infection or that can be used for monitoring disease progression or treatment response are currently still lacking. Several efforts have therefore been undertaken to identify novel biomarkers that hold potential to be used in such diagnostic tests (Vlaminck et al. 2015). For Dirofilaria immitis (heartworm) and Wuchereria bancrofti (the most prevalent cause of lymphatic filariasis), this has been successful as sensitive assays have been developed that detect circulating filarial antigens in the blood (More and Copeman 1990; Weil 1987; Weil et al. 2013; Weil et al. 1997). An approach that has shown promise is the use of metabolome analysis of serum or urine samples from infected individuals, which has led to the identification of urinary $N$-acetyltyramine- $O, \beta$-glucuronide (NATOG) as a unique biomarker for $O$. volvulus infection (Denery et al. 2010; Globisch et al. 2013; Lagatie et al. 2016).

Recent deep sequencing work has revealed the presence of many microRNA (miRNA) homologues in nematode parasites. Studies on whole worm extracts have identified miRNAs produced by Brugia malayi (Poole et al. 2010; Poole et al. 2014) and D. immitis (Fu et al. 2013). Other works suggest that these parasite miRNAs may play a role in adaptation to life in the vertebrate host (Britton et al. 2014) and possibly also modulate the innate immunity of the host (Buck et al. 2014). Indeed, deep sequencing studies identified several parasitic miRNAs in host circulation upon infection with Loa loa (Tritten et al. 2014b), Onchocerca ochengi (Tritten et al. 2014b), Litomosoides sigmodontis (Buck et al. 2014), Schistosoma mansoni (Hoy et al. 2014), D. immitis (Tritten et al. 2014a), and O. volvulus (Quintana et al. 2015; Tritten et al. 2014a).

$O$. volvulus miRNAs have been detected in serum of infected individuals (Quintana et al. 2015). Of the six different miRNAs detected (miR-71, lin-4, miR-100d, miR-87-3p, mir100a, and bantam-a), only miR-71 and lin-4 were found both in samples of patients from Cameroon and in samples of patients from Ghana. MiR-87-3p and miR-100a were only detected in samples of Ghanaian patients. The other two miRNAs (miR100d and bantam-a) were found both in infected and uninfected patients from Ghana. This work was performed only with pooled serum using small RNA sequencing on the Illumina platform. No information was collected on the detection frequency of these miRNAs in serum of infected individuals, nor on the levels in individual samples. Also, no confirmation of these findings using standard PCR technology has been performed. Therefore, we wanted to determine the levels of several (putative) $O$. volvulus miRNAs in different sets of $O$. volvulus-infected individuals using Locked Nucleic Acid (LNA)-based RT-qPCR.

\section{Materials and methods}

\section{Study samples}

The following material was obtained through the Filariasis Research Reagent Resource Center (FR3), Division of Microbiology and Infectious Diseases, NIAID, NIH: a set of 20 O. volvulus-infected human serum samples, from Dr. Nutman, collected in Cameroon. All samples were deidentified before they were provided to FR3, and usage of these samples for research purposes was approved by the Smith College Institutional Review Board (IRB). Information on $O$. volvulus infection (number of microfilaria/mg skin and number of palpable nodules) was provided by FR3, along with demographic information (Table 1). All infected individuals had at least two palpable nodules and $25 \mathrm{mf} / \mathrm{mg}$ skin (microfilaridermia) as determined by skin snip. Sera were collected from clotted blood obtained by venipuncture. The negative panel was composed of eight serum samples from healthy, non-infected individuals from Southern Africa, was collected in FDA-regulated donor centers in the USA, and was provided by Tissue Solutions Ltd. (Glasgow, Scotland). Written informed consent was obtained from all individuals, and all samples were decoded and de-identified before they were provided for research purposes. Demographic information was also provided (Table 1). All samples were stored at $-80^{\circ} \mathrm{C}$ until analysis.

A second set of EDTA plasma samples was collected as part of a field study in Ghana. This study was undertaken in an onchocerciasis-endemic community located in Adansi South District along the Pra River basins in the Ashanti Region of Ghana. This study was approved by the Committee on Human Research Publications and Ethics of the School of Medical Sciences of the Kwame Nkrumah University of Science and Technology, Kumasi, Ghana, and study subjects signed an informed consent form. Physical examinations were performed to identify those subjects having palpable nodules. Skin snips (biopsies) were then taken from those with nodules in order to determine the microfilarial (mf) load in the skin (Debrah et al. 2015). Most subjects were participating in MDA programs. A total of 23 nodule-positive subjects that donated plasma samples were included. Additionally, EDTA plasma samples from six non-endemic controls (from Kumasi, Ashanti Region) were included. An overview of the patient demographics is also provided in Table 1. All samples were stored at $-80{ }^{\circ} \mathrm{C}$ until analysis.

\section{Synthetic microRNA molecules and generation of miRNA standard curves}

Six RNase-free 5'-phosphorylated miRNA oligoribonucleotides were synthesized (Integrated DNA Technologies) for the validation of the miRNA assays, corresponding to bma-miR-71, ovmiR-71 22nt, ov-miR-71 23nt, ov-miR-87-3p, hsa-miR-425-5p, 
Table 1 Demographic information of study populations

\begin{tabular}{|c|c|c|c|c|c|c|c|c|}
\hline Subject ID & Origin & Group & Age & Sex & $\begin{array}{l}\mathrm{mf} / \mathrm{mg} \\
\text { skin }\end{array}$ & $\begin{array}{l}\text { No. of } \\
\text { nodules }\end{array}$ & $\begin{array}{l}\text { Ov16 } \\
\text { IgG4 }\end{array}$ & Source \\
\hline $852-005$ & Ghana & Nod. & 54 & M & 0 & 2 & - & KCCR \\
\hline $854-036$ & Ghana & Nod. & 62 & M & 0 & 1 & + & $\mathrm{KCCR}$ \\
\hline $853-043$ & Ghana & Nod. & 74 & M & 0 & 1 & + & $\mathrm{KCCR}$ \\
\hline $855-037$ & Ghana & Nod. & 55 & M & 0 & 2 & - & $\mathrm{KCCR}$ \\
\hline $850-011$ & Ghana & Nod. & 21 & F & 0 & 2 & + & KCCR \\
\hline $850-034$ & Ghana & Nod. & 22 & M & 0 & 1 & - & KCCR \\
\hline $852-025$ & Ghana & Nod. & 28 & F & 0 & 1 & - & KCCR \\
\hline $852-046$ & Ghana & Nod. & 25 & F & 0 & 1 & + & KCCR \\
\hline 854-009 & Ghana & Nod. & 58 & M & 0 & 1 & + & $\mathrm{KCCR}$ \\
\hline $851-033$ & Ghana & Nod. & 30 & M & 0 & 1 & + & $\mathrm{KCCR}$ \\
\hline $855-040$ & Ghana & Nod. & 50 & $\mathrm{~F}$ & 0 & 3 & + & $\mathrm{KCCR}$ \\
\hline $853-060$ & Ghana & Nod. & 44 & M & 0 & 2 & + & $\mathrm{KCCR}$ \\
\hline $854-030$ & Ghana & Nod. & 40 & F & 0 & 2 & - & $\mathrm{KCCR}$ \\
\hline $850-052$ & Ghana & Nod. & 29 & $\mathrm{~F}$ & 0 & 1 & + & $\mathrm{KCCR}$ \\
\hline $856-020$ & Ghana & Nod. & 52 & M & 0 & 5 & + & $\mathrm{KCCR}$ \\
\hline 851-014 & Ghana & Nod. & 58 & M & 0 & 2 & + & KCCR \\
\hline $853-051$ & Ghana & Nod. & 58 & $\mathrm{~F}$ & 0 & 1 & - & KCCR \\
\hline $852-028$ & Ghana & Nod. & 80 & $\mathrm{~F}$ & 0 & 1 & + & KCCR \\
\hline $852-038$ & Ghana & Nod. & 21 & M & 0 & 1 & - & KCCR \\
\hline $854-031$ & Ghana & Nod. & 60 & $\mathrm{~F}$ & 0 & 1 & + & KCCR \\
\hline 854-043 & Ghana & Nod. & 67 & M & 0.6 & 4 & + & KCCR \\
\hline 853-014 & Ghana & Nod. & 21 & M & 0 & 1 & + & KCCR \\
\hline $850-072$ & Ghana & Nod. & 28 & M & 0 & 1 & + & KCCR \\
\hline $500-501$ & Ghana & NEC & 26 & M & 0 & 0 & - & KCCR \\
\hline $500-503$ & Ghana & NEC & 25 & $\mathrm{~F}$ & 0 & 0 & - & $\mathrm{KCCR}$ \\
\hline $500-504$ & Ghana & NEC & 43 & M & 0 & 0 & - & KCCR \\
\hline $500-505$ & Ghana & NEC & 26 & M & 0 & 0 & - & KCCR \\
\hline $500-506$ & Ghana & NEC & 28 & F & 0 & 0 & - & $\mathrm{KCCR}$ \\
\hline $500-507$ & Ghana & NEC & 28 & M & 0 & 0 & - & KCCR \\
\hline MC 179 & Cameroon & $\mathrm{mf}$ & 47 & M & 100 & 7 & + & FR3 \\
\hline MC 202 & Cameroon & $\mathrm{mf}$ & 52 & M & 89 & 9 & + & FR3 \\
\hline MC 211 & Cameroon & $\mathrm{mf}$ & 54 & $\mathrm{~F}$ & 36 & 2 & + & FR3 \\
\hline MC 215 & Cameroon & $\mathrm{mf}$ & 55 & $\mathrm{~F}$ & 45 & 2 & - & FR3 \\
\hline MC 224 & Cameroon & $\mathrm{mf}$ & 60 & $\mathrm{~F}$ & 26 & 3 & + & FR3 \\
\hline MC 226 & Cameroon & $\mathrm{mf}$ & 75 & $\mathrm{~F}$ & 99 & 7 & + & FR3 \\
\hline MC 234 & Cameroon & $\mathrm{mf}$ & 50 & $\mathrm{~F}$ & 70 & 2 & + & FR3 \\
\hline MC 253 & Cameroon & $\mathrm{mf}$ & 54 & M & 40 & 6 & + & FR3 \\
\hline MC 260 & Cameroon & $\mathrm{mf}$ & 39 & $\mathrm{~F}$ & 26 & 2 & + & FR3 \\
\hline MC 319 & Cameroon & $\mathrm{mf}$ & 60 & M & 40 & 2 & + & FR3 \\
\hline MC 326 & Cameroon & $\mathrm{mf}$ & 46 & M & 45 & 2 & + & FR3 \\
\hline MC 328 & Cameroon & $\mathrm{mf}$ & 37 & M & 74 & 6 & + & FR3 \\
\hline MC 331 & Cameroon & $\mathrm{mf}$ & 54 & M & 78 & 5 & + & FR3 \\
\hline MC 333 & Cameroon & $\mathrm{mf}$ & 43 & M & 300 & 6 & + & FR3 \\
\hline MC 335 & Cameroon & $\mathrm{mf}$ & 23 & M & 30 & 4 & + & FR3 \\
\hline MC 341 & Cameroon & $\mathrm{mf}$ & 22 & M & 52 & 5 & + & FR3 \\
\hline MC 343 & Cameroon & $\mathrm{mf}$ & 35 & M & 200 & 2 & - & FR3 \\
\hline MC 352 & Cameroon & $\mathrm{mf}$ & 60 & M & 200 & 14 & + & FR3 \\
\hline MC 360 & Cameroon & $\mathrm{mf}$ & 66 & $\mathrm{~F}$ & 98 & 6 & + & FR3 \\
\hline MC 362 & Cameroon & $\mathrm{mf}$ & 70 & F & 26 & 6 & + & FR3 \\
\hline
\end{tabular}


Table 1 (continued)

\begin{tabular}{|c|c|c|c|c|c|c|c|c|}
\hline Subject ID & Origin & Group & Age & Sex & $\begin{array}{l}\mathrm{mf} / \mathrm{mg} \\
\text { skin }\end{array}$ & $\begin{array}{l}\text { No. of } \\
\text { nodules }\end{array}$ & $\begin{array}{l}\text { Ov16 } \\
\text { IgG4 }\end{array}$ & Source \\
\hline Ab-E11955 & $\begin{array}{r}\text { Southern } \\
\text { Africa }\end{array}$ & $\mathrm{HC}$ & 17 & $\mathrm{~F}$ & 0 & 0 & - & TS \\
\hline Ab-E11962 & $\begin{array}{r}\text { Southern } \\
\text { Africa }\end{array}$ & $\mathrm{HC}$ & 17 & M & 0 & 0 & - & TS \\
\hline Ab-E11968 & $\begin{array}{r}\text { Southern } \\
\text { Africa }\end{array}$ & $\mathrm{HC}$ & 17 & F & 0 & 0 & - & TS \\
\hline Ab-E11969 & $\begin{array}{r}\text { Southern } \\
\text { Africa }\end{array}$ & $\mathrm{HC}$ & 17 & $\mathrm{~F}$ & 0 & 0 & - & TS \\
\hline Ab-E11970 & $\begin{array}{r}\text { Southern } \\
\text { Africa }\end{array}$ & $\mathrm{HC}$ & 17 & $\mathrm{~F}$ & 0 & 0 & - & TS \\
\hline Ab-E12152 & $\begin{array}{r}\text { Southern } \\
\text { Africa }\end{array}$ & $\mathrm{HC}$ & 25 & M & 0 & 0 & - & TS \\
\hline Ab-E12153 & $\begin{array}{r}\text { Southern } \\
\text { Africa }\end{array}$ & $\mathrm{HC}$ & 33 & M & 0 & 0 & - & TS \\
\hline Ab-E12160 & $\begin{array}{r}\text { Southern } \\
\text { Africa }\end{array}$ & $\mathrm{HC}$ & 47 & M & 0 & 0 & - & TS \\
\hline
\end{tabular}

Nod., nodule positive, $N E C$ non-endemic control, $m f$ microfilaridermic, $H C$ healthy control, $K C C R$ Kumasi Centre for Collaborative Research, FR3 Filariasis Research Reagent Resource Center, TS Tissue Solutions, Ltd.

and hsa-miR-93-5p. Stock solutions of $100 \mu \mathrm{M}$ synthetic oligonucleotide in RNase-free and DNase-free water were prepared according to the quantities quoted by the manufacturer (based on spectrophotometric analysis). Tenfold dilution series were prepared in RNase-free and DNase-free water, starting at $10^{8}$ copies $/ \mu \mathrm{L}$ down to $10^{2}$ copies $/ \mu \mathrm{L}$. These samples and no template control (NTC; zero copies) were further processed and analyzed similar to the study samples.

\section{miRNA analysis by miRCURY LNA Universal RT miRNA PCR}

RNA was purified from 25 or $200 \mu \mathrm{L}$ serum/plasma, as indicated, with the miRNeasy kit from Qiagen (Venlo, Holland) according to the manufacturers' protocol. Reverse transcription reactions were performed using the miRCURY LNA Universal RT MicroRNA PCR Universal complementary DNA (cDNA) kit II (Exiqon, Vedbaek, Denmark). Reverse transcription thermocycling parameters were as follows: $42{ }^{\circ} \mathrm{C}$ for $60 \mathrm{~min}$ and $95^{\circ} \mathrm{C}$ for $5 \mathrm{~min}$. qPCR was performed using the miRCURY LNA Universal RT microRNA PCR system (Exiqon, Vedbaek, Denmark) with 17 parasitic miRNAs and 2 human miRNAs as internal control. All primer/probe sets for parasitic miRNAs were custom designed by the supplier. Also, three extraction controls and two cDNA synthesis controls were used as indicated by the provider. All miRNAs analyzed are listed in Table 2. Two real-time qPCR amplifications were performed for each RT reaction. Reactions were performed according to the manufacturers' instructions using a LightCycler 480 system (Roche). qPCR thermocycling conditions were as follows: $95^{\circ} \mathrm{C}$ for $10 \mathrm{~min}$, followed by 45 cycles of $95^{\circ} \mathrm{C}$ for $10 \mathrm{~s}$ and $60{ }^{\circ} \mathrm{C}$ for $1 \mathrm{~min}$. Melt curve analysis was performed between 60 and $95^{\circ} \mathrm{C}$ at a ramp rate of $0.11^{\circ} \mathrm{C} / \mathrm{s}$. Detectable miRNAs were those with a $\mathrm{Cq}$ (quantification cycle) $<40.0$ in one of both duplicates. An overview of the different sample volumes used for extraction, RNA volumes used for cDNA synthesis, and the different cDNA dilutions used for qPCR is presented in Table 3.

\section{Statistical analysis}

Results of internal and external controls (extraction controls, cDNA synthesis controls, and human reference miRNAs) were used as quality control of the samples evaluated. Results of these controls were subjected to outlier testing in order to identify samples with poor extraction and/or cDNA synthesis efficiency. For each control assay, all samples analyzed under the same conditions (i.e., same input volume and cDNA dilution) were subjected to outlier detection with a Grubbs' test (using a significance level of 0.05) using the GraphPad online outlier calculator (http://graphpad.com/quickcalcs/Grubbs1.cfm) (Grubbs 1969). In case an outlier was detected, sample was not included for further analysis. This process of outlier identification was iterated until no outliers were detected. For comparison of Tm values in different groups, two-tailed unpaired $t$ test was performed using the GraphPad Prism version 6.02. $P$ value $<0.05$ was considered to be significantly different.

\section{Results}

\section{Assay validation: standard curves and cutoff determination}

In order to confirm the performance of the miRCURY LNA Universal RT microRNA PCR system, dilution curves were 
Table 2 miRNAs analyzed in this study

\begin{tabular}{|c|c|c|c|}
\hline miRNA & Sequence & Remark & Ref. \\
\hline bma-miR-36a & TCACCGGGTGCACATTCGGTC & Female specific & Poole et al. (2014) \\
\hline bma-miR-84 & TGAGGTAGTTTATAAAGCTGCGA & Adult specific & Poole et al. (2014) \\
\hline bma-miR-5364 & CGAGGTATTGTTTATTGGCTGA & Adult specific & Poole et al. (2014) \\
\hline bma-miR-236-1 & TAATACTGTCAGGTAATGACGAT & Adult specific & Poole et al. (2014) \\
\hline bma-miR-71 21nt & TGAAAGACATGGGTAGTGAGA & mf specific & Poole et al. (2014) \\
\hline ov-miR-71 23nt & TGAAAGACATGGGTAGTGAGACG & Found in human serum & Quintana et al. (2015) \\
\hline ov-miR-71 22nt & TGAAAGACATGGGTAGTGAGAC & Found in human serum & Quintana et al. (2015) \\
\hline 1s-miR-86 & TAAGTGAATGCTTTGCCACAGTCT & Found in mouse serum & Buck et al. (2014) \\
\hline 1s-miR-263 & AATGGCACTAGATGAATTCACGG & Found in mouse serum & Buck et al. (2014) \\
\hline 1s-miR-100a/ov-miR-100a & TACCCGTAGCTCCGAATATGTGT & Found in mouse and human serum & Buck et al. (2014); Quintana et al. (2015) \\
\hline ls-miR-100b/ov-miR-100d & AACCCGTAGTTTCGAACATGTGT & Found in mouse and human serum & Buck et al. (2014); Quintana et al. (2015) \\
\hline 1s-miR-100c & AACCCGTAGAATTGAAATCGTGT & Found in mouse serum & Buck et al. (2014) \\
\hline ov-miR-87-3p & GTGAGCAAAGTTTCAGGTGTTC & Found in human serum & Quintana et al. (2015) \\
\hline 1s-bantam-a/ov-bantam-a & TGAGATCATTGTGAAAGCTATT & Found in mouse and human serum & Buck et al. (2014; Quintana et al. (2015) \\
\hline ls-bantam-b & TGAGATCACGTTACATCCGCCT & Found in mouse serum & Buck et al. (2014) \\
\hline ls-bantam-c & TGAGATCATGCCACATCCGTCT & Found in mouse serum & Buck et al. (2014) \\
\hline ov-lin-4 & TCCCTGAGACCTCTGCTGCGA & Found in human serum & Quintana et al. (2015) \\
\hline hsa-miR-425-5p & AATGACACGATCACTCCCGTTGA & & \\
\hline hsa-miR-93-5p & CAAAGTGCTGTTCGTGCAGGTAG & & \\
\hline UnsiSp2 & Extraction spike-in & & \\
\hline UnsiSp4 & Extraction spike-in & & \\
\hline UnsiSp5 & Extraction spike-in & & \\
\hline UnsiSp6 & cDNA synthesis spike-in & & \\
\hline cel-miR-39-3p & cDNA synthesis spike-in & & \\
\hline
\end{tabular}

prepared for four parasitic miRNAs (bma-miR-71, ov-miR-71 22nt, ov-miR-71 23nt, and ov-miR-87-3p) and two human reference miRNAs (hsa-miR-425-5p and hsa-miR-93-5p). Each dilution curve was analyzed in duplicate using its respective assay, and resulting $\mathrm{Cq}$ values were used to generate standard curves (Fig. 1). All tested assays had good linearity $\left(r^{2}>0.98\right)$ and acceptable PCR efficiency (slope was between -3.12 and -3.89 , corresponding to PCR efficiencies between 80.8 and $109 \%$ ). The lowest concentration used for each miRNA, 28 copies/reaction (1.45 log copies/reaction), was well detectable in all assays. The average $\mathrm{Cq}$ value for the four $O$. volvulus miRNAs at this concentration, being 35.9, was therefore chosen as cutoff for positivity in all miRNA assays used in this work. This cutoff approximates the cutoff of 37, recommended by the supplier. Based on this cutoff, for each experimental setup, a limit of detection (number of copies/ $\mu \mathrm{L}$ plasma) was calculated and ranged between 0.85 and $2.46 \log$ copies/ $\mu \mathrm{L}$ plasma (Table 3).

\section{Determination of plasma levels of parasitic miRNAs in samples from nodule-positive subjects}

Based on the information that has been published before, a list of 17 parasitic miRNAs was compiled (Buck et al. 2014;
Table 3 Variable RNA input and cDNA dilutions used for different sample sets

\begin{tabular}{lllll}
\hline Sample set & Sample input $(\mu \mathrm{L})$ & RNA input $(\mu \mathrm{L})$ & cDNA dilution & LOD $^{\mathrm{a}}(\log$ copies $/ \mu \mathrm{L}$ plasma $)$ \\
\hline KCCR & 200 & 5 & $1 / 50$ & 1.64 \\
KCCR & 200 & 5 & $1 / 8$ & 0.85 \\
FR3 + TS & 25 & 6 & $1 / 50$ & 2.46 \\
\hline
\end{tabular}

KCCR Kumasi Centre for Collaborative Research, FR3 Filariasis Research Reagent Resource Center, TS Tissue Solutions, Ltd.

${ }^{\mathrm{a}}$ Based on 28 copies/qPCR reaction and $100 \%$ extraction and cDNA synthesis efficiency 


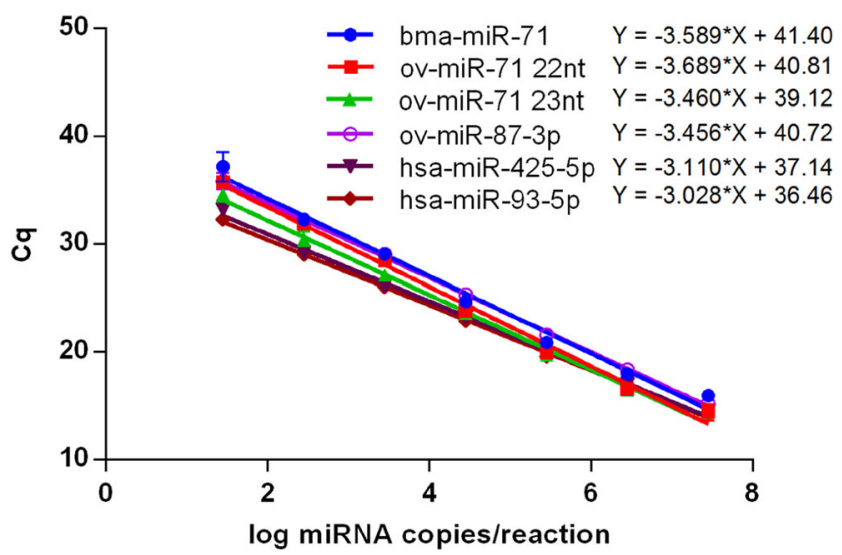

Fig. 1 Standard curves of six synthetic miRNAs

Poole et al. 2014; Quintana et al. 2015). A number of these miRNAs were found upon small RNA sequencing of serum samples from $O$. volvulus-infected individuals, while others were detected for other filarial species and have homologous sequence in $O$. volvulus (Table 2). For each of these miRNAs, a PCR primer set was designed (Exiqon, Vedbaek, Denmark) and used to analyze a set of plasma samples collected from 23 nodule-positive individuals and 6 non-endemic controls living in Ghana.

Results of internal and external controls were similar for all samples (Fig. 2a). Of the 17 parasitic miRNAs analyzed, only 5 appeared to be found positive in a number of samples: bma-miR-236-1, bma-miR-71, ov-miR-71-23nt, ov-miR$100 \mathrm{~d}$, and ov-miR-87-3p (Fig. 2b). Since it is known that less diluted cDNA is well tolerated in the miRCURY LNA Universal RT microRNA PCR system, we investigated whether sensitivity of the qRT-PCR assays could be increased by using less diluted cDNA (Egidi et al. 2015; Li et al. 2014). Therefore, samples were reanalyzed for the three miR-71 variants, ov-miR-100d, and ov-miR-87-3p, using 8fold diluted cDNA (Fig. 2c). Most samples now appeared to show positive signals for bma-miR-71, ov-miR-71-22nt, and ov-miR-71-23nt. For ov-miR-87-3p, 2 samples turned out to be positive, one more than using more diluted cDNA. Of the two positive results found for ov-miR-100d, only one could be confirmed. In order to confirm the specificity of the signals observed for the miR-71 variants and ov-miR-87-3p, melting curves obtained with the synthetic miRNAs and all clinical samples that had signal in qRT-PCR (also those with $\mathrm{Cq}$ above the cutoff) were compared (Fig. 2d). Even though both sample sets were analyzed using the same qPCR conditions, a highly significant difference in Tm values was appreciated $(P<0.001)$, indicating that the signals obtained for these miRNAs in the clinical samples are not specific and are presumably caused by non-specific amplification of one or more other miRNAs present in the samples. Results are shown for cDNA that was diluted 8-fold, but melting temperatures obtained using 50-fold diluted cDNA showed similar differences in Tm values (data not shown). In contrast, Tm values obtained for the human reference miRNAs did perfectly match between the synthetic miRNAs and clinical samples $(P>0.05)$.

\section{Determination of serum levels of parasitic miRNAs in samples from microfilaridermic subjects}

We also determined the levels of these miRNAs in serum from 20 individuals with high microfilarial load (>25 mf/ $\mathrm{mg}$ ) and 6 healthy controls. All individual results of internal and external controls are graphically summarized in Fig. 3a. Based on the different spike-ins, six samples from infected individuals out of 20 were excluded from further analysis as they showed a markedly poorer extraction efficiency and/or cDNA synthesis efficiency and were identified as outliers (samples MC260, MC211, MC224, MC234, MC253, and MC215). These samples also had lower levels of human miRNAs, confirming their poor extraction and/or cDNA synthesis efficiency. All samples from healthy volunteers had good extraction and cDNA synthesis efficiency, as well as relatively high expression of human miRNAs. When comparing the results of the human miRNAs in these serum samples with the plasma samples described previously, a substantial difference is observed. This is first of all attributed to the fact that lower input volumes have been used during extraction of these serum samples (Table 3 ). Secondly, it is known that plasma and serum miRNA levels do not fully correlate and plasma levels tend to be slightly higher (according to the guidelines for the miRCURY LNA Universal RT microRNA PCR system).

Results of the parasitic miRNAs of all samples that passed previous selection are presented in Fig. 3b. Only 4 of the 17 parasitic miRNAs investigated showed a positive signal in one of the samples analyzed (ov-bantam-a, ovmiR-71-23nt, ov-miR-100d, and ov-miR71-22nt). For the other parasitic miRNAs, signals were detected, but signals observed were very low and, in many cases, were present both in samples from infected individuals and healthy volunteers.

\section{Discussion}

The discovery of miRNAs being secreted by parasites into the circulation of the host has resulted in several speculations about their diagnostic utility. Whereas for blood-borne parasites, such as Lo. loa, D. immitis, and Li. sigmodontis, large number of different miRNAs was detected in the host circulation, initial studies on plasma or serum from $O$. volvulusinfected individuals only revealed a very small number of parasite-derived miRNAs (Buck et al. 2014; Quintana et al. 2015; Tritten et al. 2014a; Tritten et al. 2014b). Also, the 
a

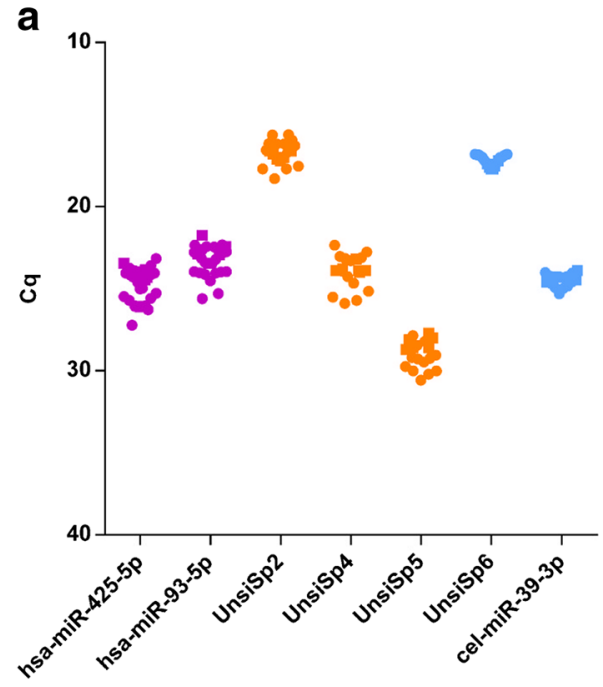

C

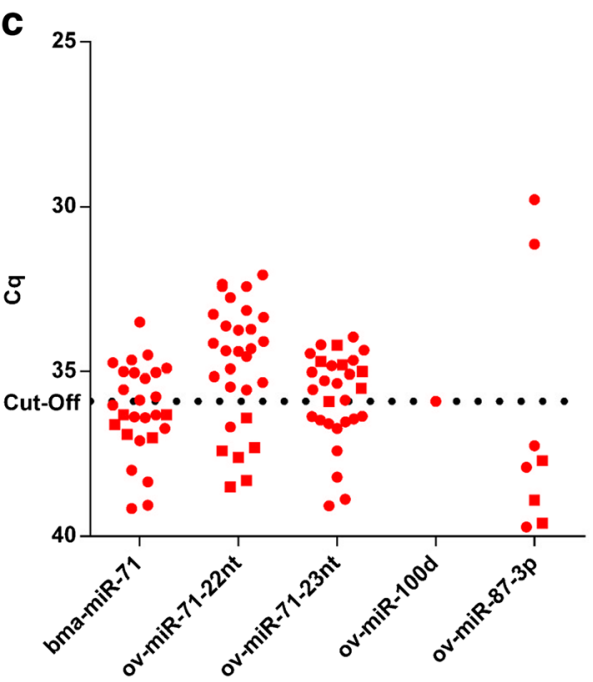

Fig. 2 miRNA analysis in plasma from nodule-positive individuals (closed circles) and non-endemic controls (closed squares) from Ghana. a Internal and external control results. b Results of 17 parasitic miRNAs.

amount of these miRNAs was very low, already indicating a sensitivity challenge for its diagnostic utility. This sensitivity challenge becomes even more important when looking to the possibility of using parasite-derived miRNAs for treatment monitoring, as a sufficiently broad dynamic range is required for this application.

We have used standard miRNA extraction and RT-qPCR technology to detect 17 of these previously identified miRNAs in plasma or serum from $O$. volvulus-infected individuals. A first sample set was collected from nodule-positive individuals in Ghana with very low or no microfilaria detected in the skin snip, while a second sample set was obtained from nodule-positive individuals in Cameroon with high microfilarial load. When both datasets are combined, only seven miRNAs appeared to result in a detectable signal in qPCR: b
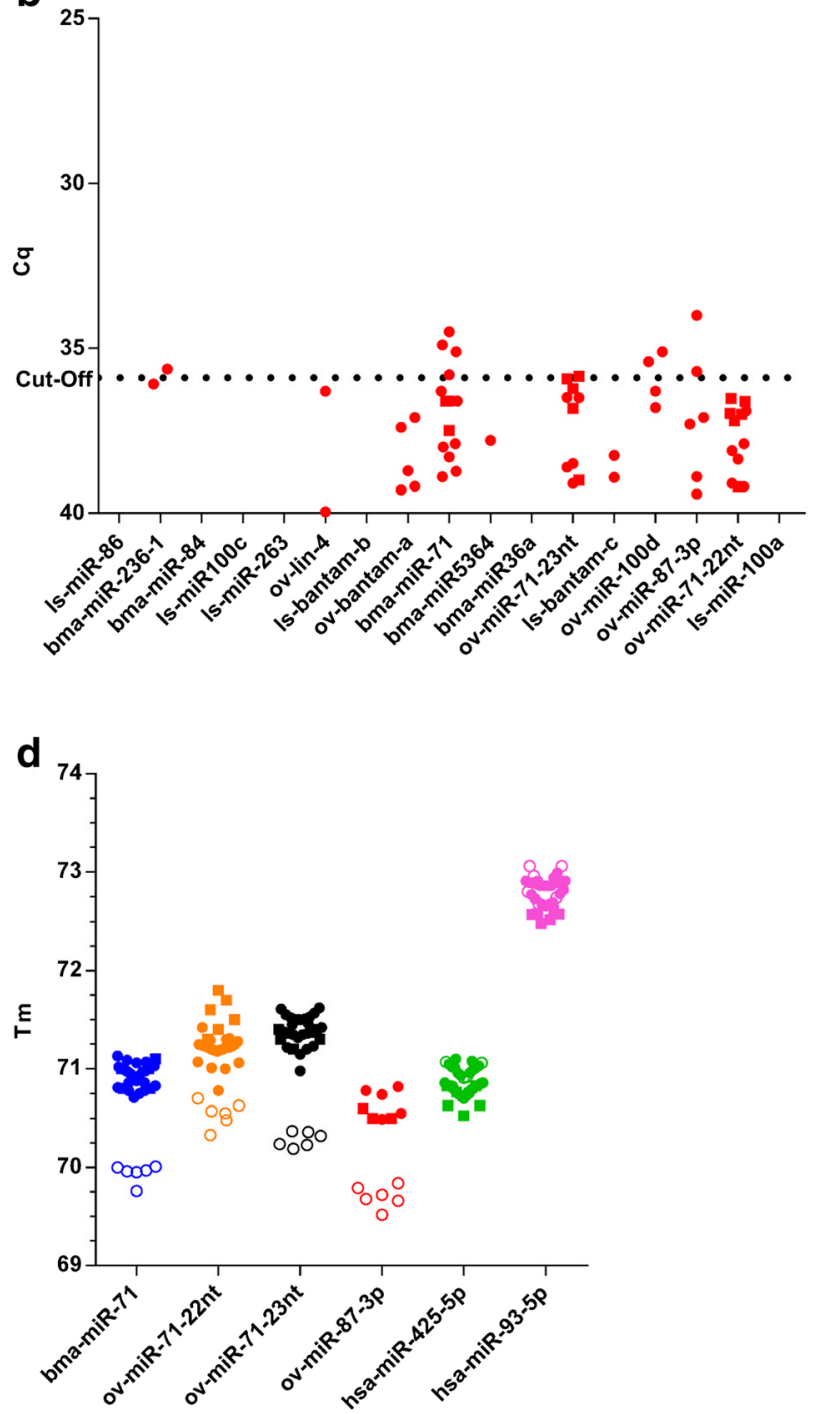

c Result of five selected $O$. volvulus miRNAs, using less diluted cDNA. d Tm determination of miRNA amplicons obtained with clinical samples (closed circles) and synthetic miRNAs (open circles)

bma-miR-236-1, bma-miR-71, ov-miR71-22nt, ov-miR-7123nt, ov-miR-100d, ov-bantam-a, and ov-miR-87-3p. Interestingly, all except bma-miR-236-1 were previously also detected in serum from infected individuals (Quintana et al. 2015). For four of these parasitic miRNAs (three miR-71 variants and ov-miR-87-3p), calibration curves were prepared using synthetic miRNAs and Tm was determined both for these synthetic miRNAs and the clinical samples. For all four parasitic miRNAs, Tm differed significantly between the clinical samples and the synthetic miRNAs, while a similar exercise for the human reference miRNAs showed no difference. This observation strongly suggests that the signals observed for these parasitic miRNAs are caused by non-specific amplification and are not reflecting true positive signals. Furthermore, as was also observed previously, plasma levels 
a

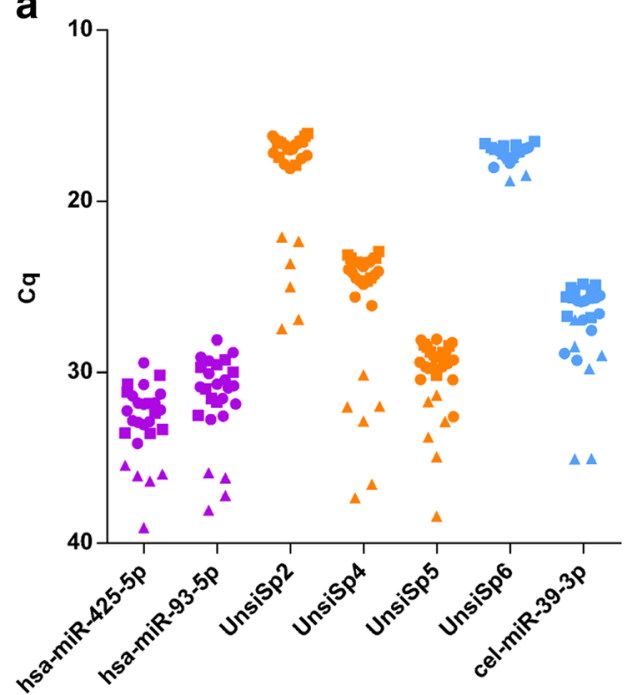

b

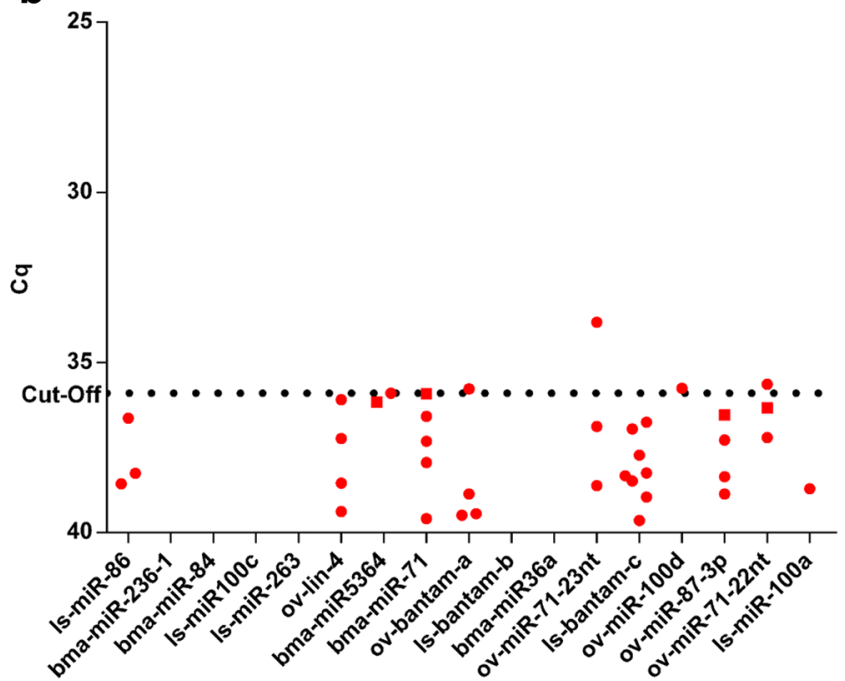

Fig. 3 miRNA analysis in serum from individuals with high microfilarial load from Cameroon (closed circles) and healthy controls from Southern Africa (closed squares). a Internal and external control results. Samples that showed reduced extraction and/or cDNA synthesis are indicated by triangles. b Results of 17 parasitic miRNAs of the other miRNAs are extremely low with Cq values just below the cutoff. Also, these miRNAs were detected in only very few samples and are not universally present in all or most infected individuals, which is an essential property for use as a diagnostic marker.

The discrepancy in Tm between synthetic miRNAs and clinical samples highlights the importance of using synthetic miRNAs to confirm the specificity of the RT-qPCR signals obtained. Whereas in small RNA sequencing technologies, the sequence of every single miRNA molecule is determined, qPCR-based detection methodologies are sensitive to nonspecific detection (Dellett and Simpson 2016). Since miRNAs are usually only 18-22 nucleotides in length, specificity remains one of the key issues when developing RTqPCR assays to measure microRNAs for molecular diagnosis (Bartel 2004; Dellett and Simpson 2016). Especially when analyzing low abundant miRNAs in a background of millions of copies of other miRNAs (and other small RNAs), one needs to confirm the specificity of the assays before drawing any conclusion.

Importantly, our study does not completely exclude the applicability of plasma-derived parasitic miRNAs as a tool to detect the presence of parasite using minimally invasive sampling procedures. Our work indicates that sensitive methodologies will be required that also demonstrate high specificity. As this study was limited to only one qPCR-based technology using commercially available reagents, further optimization of the qPCR assays or evaluation of other detection platforms, such as stem-loop RT-PCR, might offer some improvements (Chen et al. 2005; Mestdagh et al. 2014). It remains, however, questionable whether detection of parasitic miRNAs with sufficient diagnostic power will be feasible, even after elaborate assay optimization. The most obvious path forward to increase sensitivity might be to use higher volumes of plasma for miRNA extraction. Standard miRNA extraction kits, however, are not designed to use higher volumes, and this approach would therefore have limited feasibility in practice. Alternatively, a preamplification step might be introduced as this has been shown before to boost sensitivity (Tan et al. 2015). One other interesting approach might be the use of a precursory isolation of parasite-specific exosomes. It was shown for the helminth parasites Heligmosomoides polygyrus and B. malayi that these parasites secrete miRNAcontaining vesicles, possibly involved in modulation of the host's immune response (Buck et al. 2014; Zamanian et al. 2015; Zheng et al. 2016). Isolation of total exosomes or even of parasite-specific exosomes could result in an enrichment of parasitic miRNAs, thereby reducing the background of nonparasite-derived miRNAs during analysis. The same work, however, also indicated that exosomes and associated RNAs operate locally in the host's body and that their detection will depend on the stage and localization of the parasite in the host (Buck et al. 2014). It is therefore not surprising to observe the low amounts of parasitic miRNAs in the circulation upon infection with the skin localized $O$. volvulus as compared to, e.g., infection with $D$. immitis or $B$. malayi, two parasites with a blood-borne microfilarial stage (Tritten et al. 2014a).

In conclusion, our data indicate that $O$. volvulus miRNAs are not present in circulation in sufficiently high amounts to be used as a diagnostic marker for infection or treatment monitoring using LNA-based RT-qPCR analysis.

Cq, Quantification cycle; mf, microfilaria; miRNA, microRNA; O. volvulus, Onchocerca volvulus; qPCR, quantitative PCR; RT, reverse transcriptase 
Acknowledgements We gratefully acknowledge the Filariasis Research Reagent Resource Center (FR3) at the Smith College, Northampton, USA, for providing serum samples from $O$. volvulus-infected individuals under a contract from the National Institute of Allergy and Infectious Diseases, USA. We also would like to thank Steven Silber for his contributions in collecting samples in Ghana. We thank Janssen Biobank for logistic support and Bieke van Dorst, Liesbeth Van Wesenbeeck, and Hanne Meeuws for scientific discussions.

Authors' contributions OL, AD, LBD, and LJS designed the study. $\mathrm{OL}$ and LBD performed the research. OL and LJS performed the analysis of the data. OL and LJS wrote the manuscript. All authors read and approved the final manuscript.

\section{Compliance with ethical standards}

Ethical approval All procedures performed in studies involving human participants were in accordance with the ethical standards of the institutional and/or national research committee and with the 1964 Helsinki Declaration and its later amendments or comparable ethical standards.

Informed consent Informed consent was obtained from all individual participants included in the study.

Conflict of interest Authors OL and LJS are current employees of Janssen Pharmaceutica NV, a Johnson and Johnson Company, and may own stock or stock options in that company.

Open Access This article is distributed under the terms of the Creative Commons Attribution 4.0 International License (http:// creativecommons.org/licenses/by/4.0/), which permits unrestricted use, distribution, and reproduction in any medium, provided you give appropriate credit to the original author(s) and the source, provide a link to the Creative Commons license, and indicate if changes were made.

\section{References}

Bartel DP (2004) MicroRNAs: genomics, biogenesis, mechanism, and function. Cell 116(2):281-297

Borup LH, Peters JS, Sartori CR (2003) Onchocerciasis (river blindness). Cutis 72(4):297-302

Britton C, Winter AD, Gillan V, Devaney E (2014) microRNAs of parasitic helminths - identification, characterization and potential as drug targets. International Journal for Parasitology-Drugs and Drug Resistance 4(2):85-94. doi:10.1016/j.ijpddr.2014.03.001

Buck AH et al (2014) Exosomes secreted by nematode parasites transfer small RNAs to mammalian cells and modulate innate immunity. Nat Commun 5:5488. doi:10.1038/ncomms6488

Chandrashekar R, Ogunrinade AF, Weil GJ (1996) Use of recombinant Onchocerca volvulus antigens for diagnosis and surveillance of human onchocerciasis. Tropical medicine \& international health : TM \& IH 1(5):575-580

Chen C et al (2005) Real-time quantification of microRNAs by stem-loop RT-PCR. Nucleic Acids Res 33(20):e179. doi:10.1093/nar/gni178

Cupp EW, Sauerbrey M, Richards F (2011) Elimination of human onchocerciasis: history of progress and current feasibility using ivermectin (Mectizan((R))) monotherapy. Acta Trop 120(Suppl 1):S100-S108. doi:10.1016/j.actatropica.2010.08.009

Debrah AY et al (2015) Doxycycline leads to sterility and enhanced killing of female Onchocerca volvulus worms in an area with persistent microfilaridermia after repeated ivermectin treatment: a randomized, placebo-controlled, double-blind trial. Clinical infectious diseases : an official publication of the Infectious Diseases Society of America 61(4):517-526. doi:10.1093/cid/civ363

Dellett M, Simpson DA (2016) Considerations for optimization of microRNA PCR assays for molecular diagnosis. Expert Rev Mol Diagn 16(4):407-414. doi:10.1586/14737159.2016.1152184

Denery JR, Nunes AA, Hixon MS, Dickerson TJ, Janda KD (2010) Metabolomics-based discovery of diagnostic biomarkers for onchocerciasis. PLoS Negl Trop Dis 4(10). doi:10.1371/journal. pntd.0000834

Egidi MG et al (2015) Stability assessment of candidate reference genes in urine sediment of prostate cancer patients for miRNA applications. Dis Markers 2015:973597. doi:10.1155/2015/973597

Enk CD (2006) Onchocerciasis - river blindness. Clin Dermatol 24(3): 176-180. doi:10.1016/j.clindermatol.2005.11.008

$\mathrm{Fu}$ Y et al (2013) Identification of Dirofilaria immitis miRNA using Illumina deep sequencing. Vet Res 44:3. doi:10.1186/1297-9716-44-3

Globisch D et al (2013) Onchocerca volvulus-neurotransmitter tyramine is a biomarker for river blindness. P Natl Acad Sci USA 110(11): 4218-4223. doi:10.1073/pnas.1221969110

Golden A et al (2013) Extended result reading window in lateral flow tests detecting exposure to Onchocerca volvulus: a new technology to improve epidemiological surveillance tools. PLoS One 8(7): e69231. doi:10.1371/journal.pone.0069231

Grubbs FE (1969) Procedures for detecting outlying observations in samples. Technometrics 11:1-21

Hoy AM et al (2014) Parasite-derived microRNAs in host serum as novel biomarkers of helminth infection. PLoS Negl Trop Dis 8(2):e2701. doi:10.1371/journal.pntd.0002701

Lagatie $\mathrm{O}$ et al (2016) Evaluation of the diagnostic potential of urinary $\mathrm{N}$ acetyltyramine-O,beta-glucuronide (NATOG) as diagnostic biomarker for Onchocerca volvulus infection. Parasit Vectors 9(1): 302. doi:10.1186/s13071-016-1582-6

Lavebratt C et al (1994) A simple dot blot assay adaptable for field use in the diagnosis of onchocerciasis: preparation of an adult worm antigen fraction which enhances sensitivity and specificity. Trans R Soc Trop Med Hyg 88(3):303-306

Li P et al (2014) Circulating microRNAs serve as novel biological markers for intracranial aneurysms. J Am Heart Assoc 3(5): e000972. doi:10.1161/JAHA.114.000972

Lipner EM et al (2006) Field applicability of a rapid-format anti-Ov16 antibody test for the assessment of onchocerciasis control measures in regions of endemicity. J Infect Dis 194(2):216221. doi: $10.1086 / 505081$

Mestdagh P et al (2014) Evaluation of quantitative miRNA expression platforms in the microRNA quality control (miRQC) study. Nat Methods 11(8):809-815. doi:10.1038/nmeth.3014

More SJ, Copeman DB (1990) A highly specific and sensitive monoclonal antibody-based ELISA for the detection of circulating antigen in bancroftian filariasis. Tropical medicine and parasitology : official organ of Deutsche Tropenmedizinische Gesellschaft and of Deutsche Gesellschaft fur Technische Zusammenarbeit 41(4):403-406

Poole CB, Davis PJ, Jin J, McReynolds LA (2010) Cloning and bioinformatic identification of small RNAs in the filarial nematode, Brugia malayi. Mol Biochem Parasitol 169(2):87-94. doi:10.1016/j.molbiopara.2009.10.004

Poole CB et al (2014) Diversity and expression of microRNAs in the filarial parasite, Brugia malayi. PLoS One 9(5):e96498. doi:10.1371/journal.pone.0096498

Quintana JF et al (2015) Extracellular Onchocerca-derived small RNAs in host nodules and blood. Parasit Vectors 8:58. doi:10.1186 /s13071-015-0656-1

Steel C et al (2015) Rapid point-of-contact tool for mapping and integrated surveillance of Wuchereria bancrofti and Onchocerca volvulus 
infection. Clinical and vaccine immunology : CVI 22(8):896-901. doi:10.1128/CVI.00227-15

Tan GW, Khoo AS, Tan LP (2015) Evaluation of extraction kits and RT-qPCR systems adapted to high-throughput platform for circulating miRNAs. Scientific reports 5:9430. doi:10.1038 /srep09430

Taylor HR, Munoz B, Keyvan-Larijani E, Greene BM (1989) Reliability of detection of microfilariae in skin snips in the diagnosis of onchocerciasis. Am J Trop Med Hyg 41(4):467471

Tritten L et al (2014a) Detection of circulating parasite-derived microRNAs in filarial infections. PLoS Negl Trop Dis 8(7):e2971. doi:10.1371/journal.pntd.0002971

Tritten L et al (2014b) Loa loa and Onchocerca ochengi miRNAs detected in host circulation. Mol Biochem Parasitol 198(1):14-17. doi:10.1016/j.molbiopara.2014.11.001

Vlaminck J, Fischer PU, Weil GJ (2015) Diagnostic tools for onchocerciasis elimination programs. Trends Parasitol 31(11):571-582. doi:10.1016/j.pt.2015.06.007
Weil GJ (1987) Dirofilaria immitis: identification and partial characterization of parasite antigens in the serum of infected dogs. Exp Parasitol 64(2):244-251

Weil GJ, Lammie PJ, Weiss N (1997) The ICT filariasis test: a rapidformat antigen test for diagnosis of bancroftian filariasis. Parasitol Today 13(10):401-404

Weil GJ et al (2000) A rapid-format antibody card test for diagnosis of onchocerciasis. J Infect Dis 182(6):1796-1799. doi:10.1086/317629

Weil GJ et al (2013) Laboratory and field evaluation of a new rapid test for detecting Wuchereria bancrofti antigen in human blood. Am J Trop Med Hyg 89(1):11-15. doi:10.4269/ajtmh.13-0089

WHO (2010) First WHO report on neglected tropical diseases: working to overcome the global impact of neglected tropical diseases

Zamanian M et al (2015) Release of small RNA-containing exosome-like vesicles from the human filarial parasite Brugia malayi. PLoS Negl Trop Dis 9(9):e0004069. doi:10.1371/journal.pntd.0004069

Zheng Y et al (2016) Effects of Echinococcus multilocularis miR-71 mimics on murine macrophage RAW264.7 cells. Int Immunopharmacol 34: 259-262. doi:10.1016/j.intimp.2016.03.015 